\title{
Neuromyelitis Optica Spectrum Disorder as a Para- neoplastic Disorder in a Patient with Metastatic Breast Cancer
}

\author{
Vargas WS, Marc Dinkin, Nancy Nealon and Perumal JS* \\ Department of Neurology and Neuroscience, Weill Cornell Medical College, USA
}

Received: February 19, 2014; Accepted: March 14, 2014; Published: March 18, 2014

*Corresponding author: Perumal JS, Department of Neurology and Neuroscience, Weill Cornell Medical College, 1305 York Ave, 2nd floor, New York, NY 10021, USA, Tel: 646-962-5733; Fax: 646-962-0390; E-mail: jsp9007@med.cornell.edu

\begin{abstract}
Neuromyelitis optica (NMO) is an inflammatory disorder of the central nervous system characterized by optic neuritis and transverse myelitis. The identification of NMO IgG ab led to the definition of NMO spectrum disorders. There have been a few reports of NMO spectrum disorders in association with cancers. The present case study describes a woman diagnosed with metastatic breast cancer and leptomeningeal spread who presented with bilateral optic neuritis and who was found to have the NMO-IgG. Reports such as this show that the potential para-neoplastic association should be considered when seeing a patient with an NMO spectrum disorder and in the appropriate clinical setting investigating an underlying cancer might be important. Studies of the diverse presentations of NMO in different clinical settings could lead to a better understanding of the underlying disease mechanisms and can potentially provide targets for therapeutic intervention.
\end{abstract}

Keywords: Neuromyelitis optica; Aquaporin for antibody; optic neuritis; Paraneoplastic syndrome

\section{Abbreviations}

NMO: Neuromyelitis optica; NMO-IgG: Neuromyelitis Optica Immunoglobulin antibody; CSF: Cerebrospinal fluid; MRI: Magnetic Resonance Imaging

\section{Introduction}

Classic NMO is defined as the simultaneous occurrence of bilateral optic neuritis and transverse myelitis [1]. NMO spectrum represents a group of neurologic disorders defined by the presence of NMO-IgG involving autoimmunity against aquaporin-4 water channel [2,3]. NMO-IgG has been incidentally discovered in patients with cancer, possibly reflecting a paraneoplastic immune response $[4,5]$. The present work describes a case of a woman diagnosed with metastatic breast cancer and leptomeningeal spread who presented with bilateral optic neuritis and was found to have the NMO-IgG.

\section{Case Report}

A 56 year old woman with a history of metastatic breast cancer presented with acute, severe, bilateral vision loss and headache. She had been diagnosed with breast cancer metastatic to the chest wall 18 months ago and had undergone lumpectomy and chemotherapy. Six months prior to current presentation, she was found to have recurrent metastatic breast cancer and had undergone right mastectomy. She refused a full course of chemotherapy secondary to side effects and had not received radiation therapy. She was being treated with Capecitabine. She also had a history of human immunodeficiency virus infection with a viral titer that was undetectable and CD-4 count of 437 $\mathrm{mm}^{3}$.

She was admitted to the hospital for evaluation of her symptoms. Visual acuity was 20/200 bilaterally with evidence of red-desaturation bilaterally. A fundoscopic exam revealed the presence of optic nerve pallor bilaterally but no edema. CSF revealed 24 white cells with a lymphocytic predominance, 1783 red cells, normal glucose and an elevated protein of 66 $\mathrm{mg} / \mathrm{dL}$. Infectious etiologies in the CSF were ruled out. CSF cytology revealed the presence of malignant cells. Brain MRI showed abnormal gadolinium enhancement of the optic chiasm, tuber cinerum and anteroinferior recess of the third ventricle consistent with leptomeningeal metastatic disease (Figure 1A, 1B). Serum NMO-IgG was positive.

She was treated with intravenous steroids without significant benefit. Unfortunately, her clinical status continued to deteriorate and she succumbed to multiple complications from

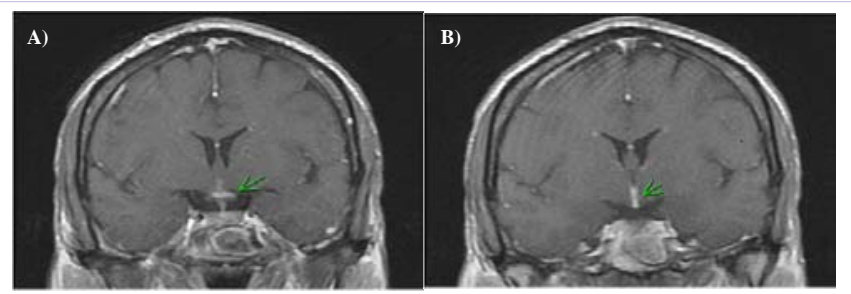

Figure 1: A) T1 coronal post contrast image demonstrates enhancement of optic chiasm. B) T1 coronal post contrast image demonstrates enhancement of third ventricle. 
extensive metastatic disease. She died one month after the onset of neurologic symptoms.

\section{Discussion}

The report describes a 56 year old woman with metastatic breast cancer who presented with bilateral optic neuritis and was found to have the NMO-IgG in her serum. Recurrent metastatic disease was detected during her presentation suggesting a potential association with the malignancy.

NMO has been previously recognized as a possible paraneoplastic phenomenon. In the earliest reported study exploring NMO-IgG in the context of cancers, there were several incidental findings of positive NMO-IgG among patients evaluated for paraneoplastic antibodies and also positive NMO-IgG in patients with known cancers where an NMO spectrum disorder was suspected [5]. NMO-IgG was associated with several cancers including breast, thyroid, carcinoid, pituitary somatotropinoma and B-cell lymphoma. Case reports have described a probable association with lung and thymic cancers and NMO as well $[5,6]$.

Several auto antibodies targeting the nervous system have been recognized as para-neoplastic phenomenon and many onconeural antigens have been identified. It is conceivable that NMO-IgG was produced as an immune response to the underlying cancer and with appropriate immune triggers lead to the clinical presentation of an NMO disorder. Another plausible explanation for the patient's presentation when she had a relapse of NMO coinciding with leptomeningeal spread is that she may have been an asymptomatic carrier of NMO-IgG and immune processes elicited by the cancer provided the trigger for the relapse. An earlier report raised this hypothesis when they described an 87 year old man who developed an NMO spectrum disorder after a diagnosis of prostate adenocarcinoma and relapsed after pneumococcal vaccination [7]. It has been demonstrated that NMO-IgG alone does initiate injury but additional factors including break down of the blood brain barrier and perhaps immune mediation by $\mathrm{T}$ cell or complement activation is implicated in the pathogenesis.

This is the first reported case of an NMO spectrum disorder in a patient with leptomeningeal carcinomatosis. It adds to accumulating evidence that NMO can present as para-neoplastic syndrome. The potential para-neoplastic association should be kept in mind when seeing a patient with an NMO spectrum disorder and in the appropriate clinical setting investigating an underlying cancer might be important. A better understanding of the underlying disease mechanisms can potentially lead to targets for therapeutic intervention.

\section{References}

1. Wingerchuk DM (2006) Neuromyelitis Optica. Int MS J 13(2): 42-50.

2. Wingerchuk DM, Lennon VA, Pittock SJ, Lucchinetti CF, Weinshenker BG (2006) Revised diagnostic criteria for neuromyelitis optica. Neurology 66(10): 1485-1489.

3. Lennon VA, Wingerchuk DM, Kryzer TJ, Pittock SJ, Lucchinetti CF, et al. (2004) A serum autoantibody marker of neuromyelitis optica: distinction from multiple sclerosis. Lancet 364(9451): 2106-2112.

4. Pittock SJ, Weinshenker BG, Wingerchuk D, et al. (2006) Autoimmune neurological accompaniments of neuromyelitis optica (NMO). Ann Neurol 60: S41.

5. Pittock SJ, Lennon VA (2008) Aquaporin-4 autoantibodies in a paraneoplastic context. Arch Neurol 65(5): 629-632.

6. De Santis G, Caniatti L, De Vito A, De Gennaro R, Granieri E, et al. (2009) A possible paraneoplastic neuromyelitis optica associated with lung cancer. Neurol Sci 30(5): 397-400.

7. Kitazawa Y, Warabi Y, Bandoh M, Takahasi T, Matsubara S (2012) Elderly-onset neuromyelitis optica which developed after the diagnosis of prostate adenocarcinoma and relapsed after a 23-valent pneumococcal polysaccharide vaccination. Intern Med 51(1): 103107. 